\title{
EL LABORATORIO FOSTER DE LA RESIDENCIA DE SEÑORITAS. LAS RELACIONES DE LA JAE CON EL INTERNATIONAL INSTITUTE FOR GIRLS IN SPAIN, Y LA FORMACIÓN DE LAS JÓVENES CIENTÍFICAS ESPAÑOLAS
}

\author{
Carmen Magallón Portolés \\ Seminario Interdisciplinar de Estudios de la Mujer (SIEM). Universidad de Zaragoza
}

\section{RESUMEN}

En el primer tercio del siglo XX crecieron diversas líneas de relación entre las universitarias norteamericanas y españolas; en particular, la que se estableció entre la Junta para Ampliación de Estudios e Investigaciones Científicas (JAE) y el International Institute for Girls in Spain (IIGS) influyó positivamente en la formación de las mujeres de ciencia españolas. Esta relación se concretó en un intercambio de estudiantes y profesoras, y en el establecimiento de una línea de becas para las jóvenes graduadas. El artículo incluye la historia del Laboratorio Foster y la biografía de su fundadora.

PALABRAS CLAVE: Primer tercio del siglo XX, JAE, Instituto Internacional, científicas españolas, Foster, Laboratorio Foster.

THE FOSTER LABORATORY IN THE RESIDENCIA DE SENORITAS. THE JAE'S RELATIONSHIP WITH THE INTERNATIONAL INSTITUTE FOR GIRLS IN SPAIN, AND THE TRAINING OF SPANISH SCIENTISTS WOMEN.

\begin{abstract}
In the first third of the twentieth century, relations among American and Spanish university women began; particularly the relationship between the JAE and the International Institute for Girls in Spain had a positive influence in the education of women scientists in Spain. An interchange of students and teachers came out from this relationship, and Spanish women received scholarships to stay in American universities. The history of the Foster Laboratory and some biographical notes of her founder are included in the paper.
\end{abstract}

KEY WORDS: First third of the twentieth century, Spanish Educational Board, International Institute for Girls in Spain, women scientists, Foster, Foster Laboratory. 


\section{LA JAE Y LA FORMACIÓN CIENTÍFICA DE LAS MUJERES}

Desde su fundación, en 1907, la Junta para Ampliación de Estudios e Investigaciones Científicas (en adelante, $\mathrm{JAE}^{1}$ ) mantuvo una disposición favorable hacia la promoción científica de las mujeres. Los hombres que la integraban, librepensadores y liberales como Giner, Cossío, Luis de Zulueta o Castillejo, respondían a los proyectos y estilo de los hombres de la Institución Libre de Enseñanza, uno de los núcleos favorables a la educación superior de las mujeres. A los que pronto se uniría la gran impulsora de la educación universitaria femenina, María de Maeztu.

María de Maeztu y Whitney², doctora en filosofía por la Universidad de Madrid, fue la única mujer que a lo largo de los años formaría parte de la JAE, al ser nombrada por Real Orden de 23 de marzo de 1928, tras la muerte de José Rodríguez Carracido, uno de los vocales fundadores. Doctora Honoris Causa por el Smith College (Northampton, Massachussets, USA) y primera presidenta de la Juventud Universitaria Femenina (precedente de la Asociación Española de Mujeres Universitarias) ${ }^{3}$, creada en 1920, María de Maeztu sería el alma de dos proyectos que impulsó la JAE, que llegaron a ser dos importantes vías de promoción de la formación superior femenina: la Residencia de Señoritas, y la concesión de becas a las jóvenes licenciadas, apoyadas por instituciones universitarias femeninas norteamericanas. De ella se dice en las Memorias de la JAE:

«La señorita de Maeztu, identificada con la obra de la Junta, a la cual ha prestado su cooperación fundando y dirigiendo la Residencia de Señoritas, ha sido la

1 Sobre la JAE, véase SuBIRÁ, José (1924) «Una gran obra de cultura patria. La Junta para Ampliación de Estudios». Nuestro tiempo, enero-abril-mayo, 1924; CASTILLEJO, José (1976) Guerra de ideas en España. Madrid, Revista de Occidente, especialmente el capítulo 11; LAPORTA, F. et al. (1987) «Los orígenes culturales de la Junta para Ampliación de Estudios». Arbor, 493, enero 1987, 17-87; LAPORTA, F. et al. (1987) «Los orígenes culturales de la Junta para Ampliación de Estudios». Arbor, 499-500, julio-agosto 1987, 9-137; MoRENO, Antonio y SÁNCHEZ RoN, J. Manuel (1987) «La Junta para Ampliación de Estudios e Investigaciones Científicas: La vida breve de una fundación ahora octogenaria», Mundo Científico, 65, enero 1987, 20-33; SÁNCHEz Ron, J. Manuel (coord.) (1988)1907-1987. La Junta para Ampliación de Estudios e Investigaciones Científicas 80 años después. Madrid, CSIC, 2 vols.

2 Sobre Maeztu, véase: PÉrez Villanueva Tovar, Isabel (1989), María de Maeztu: una mujer en el reformismo educativo español, Madrid, UNED.

3 CRESPO, Lola (1990), «La Juventud Universitaria Femenina (1920-1936)». En MAILLARD, M. Luisa, Asociación Española de Mujeres Universitarias (1920-1990), Madrid, AEMU, Instituto de la Mujer, 11-31. 
primera mujer llamada a formar parte de esta corporación. Su preparación y su experiencia en problemas pedagógicos, su prestigio y su inteligencia aumentarán considerablemente los medios de acción de la Junta» ${ }^{4}$.

La Residencia de Señoritas ${ }^{5}$, creada por la JAE en 1915, cinco años más tarde que su homóloga masculina, formaba parte de la Residencia de Estudiantes. Aunque ésta se identificó durante años exclusivamente con el grupo de varones, en lo que constituye un ejemplo de la tendencia a invisibilizar la parte de la historia que atañe a las mujeres, las Memorias de la JAE dejan claro que la Residencia de Estudiantes acogía en su seno distintos grupos:

«La Residencia de Estudiantes ofrece ya en sus varios grupos alojamientos a 250 jóvenes de uno y otro sexo (...) faltos en las escuelas oficiales de internados y de suficiente tutela cuando no viven con sus familias. Así han ido surgiendo, primero un Grupo universitario para estudiantes varones de diez y seis años en adelante, establecido ahora en la calle del Pinar; luego un Grupo de señoritas para estudiantes mujeres de igual edad, que tiene hoy sus locales en la calle Fortuny» ${ }^{6}$.

La Residencia de Señoritas se ubicó en los números 28 y 30 de la calle Fortuny, tras el traslado de la Residencia de Estudiantes varones a la calle Pinar, dando alojamiento a las alumnas que iban a estudiar a la Universidad de Madrid o preparaban su ingreso en ella, así como a las que asistían a la Escuela Superior del Magisterio, al Conservatorio Nacional de Música, la Escuela Normal, la Escuela del Hogar u otros centros de enseñanza; también a otras que privadamente se dedicaban al estudio en bibliotecas, laboratorios, archivos o clínicas. Desde su fundación, y a lo largo de su existencia, la Residencia de Señoritas estuvo siempre dirigida por María de Maeztu.

Como sucedió con la de varones, la Residencia de Señoritas contribuyó a abrir puertas y a despertar en las jóvenes de la época nuevas aspiraciones personales y profesionales. Además de dar alojamiento, disponía de servicios como biblioteca, laboratorios, clases complementarias a las de la universidad,

4 Memorias de la JAE, 1926-1928. Madrid, 1929, p. XII.

5 Sobre la Residencia de Señoritas, véase: Zulueta, Carmen de y Moreno, Alicia (1993) Ni Convento ni College. La Residencia de Señoritas. Madrid, CSIC; MAGAllón PORTOLÉs, Carmen (2001) «La Residencia de Estudiantes para Señoritas y el Laboratorio Foster. Mujeres de ciencia en España, a principios del siglo XX», ÉNDOXA, Series Filosóficas, $\mathrm{n}^{\circ} 14$, 157-181; y VÁzQUEZ RAMIL, Raquel (2001) La Institución Libre de Enseñanza y la Educación de la Mujer en España: la Residencia de Señoritas (1915-1936). (ISBN: 84-607-3148-0). Disponible en: http://webs.uvigo.es/pmayobre/colaboraciones.htm\#raquel_vazquez_ramil

6 Memorias de la JAE, 1918-19, pp. 15-16. 
cursos de idiomas, y conferencias. Allí se orientaba a las alumnas a la lectura, la asistencia a conferencias y la relación social; se llevaba con ellas una labor de tutorización, educando en libertad y en la valoración del trabajo intelectual. En los primeros años predominaban las estudiantes de Magisterio, pero con el tiempo fueron creciendo las universitarias y disminuyendo las que asistían a otros centros. Así, las residentes que estudiaban en la Universidad de Madrid pasaron de ser el 17\%, en el curso 1915-16, a ser un $71 \%$ en el curso 1933-34, último del que se disponen datos. En esta evolución se percibe la impronta de su directora, María de Maeztu, firme partidaria de opciones profesionales para las mujeres distintas a la tradicional salida de la enseñanza.

\section{PENSIONADAS POR LA JAE}

La obtención de una pensión para completar la preparación científica en el extranjero, fue para las jóvenes universitarias de la época, al igual que en el caso de los varones, el cauce para aumentar su competencia científica; una vez obtenida la formación, la mayoría fueron integrándose, codo a codo con los varones, en los incipientes equipos de investigación que estaban formándose en el país.

En 1907, a la JAE se le había encargado la tarea de concesión de pensiones o «servicio de ampliación de estudios dentro y fuera de España»; también las Delegaciones en Congresos Científicos, el servicio de información extranjera y relaciones internacionales en materia de enseñanza, el fomento de los trabajos de investigación científica y la protección de las instituciones educativas en la enseñanza secundaria y superior $\rangle^{7}$. Estas responsabilidades se concretaban en el mantenimiento de relaciones con Hispanoamérica, EEUU y otros países y la gestión de instituciones como el Patronato de Estudiantes y la Escuela Española de Arqueología e Historia de Roma, la Residencia de Estudiantes (de varones y de Señoritas), los llamados Grupo de niños y Grupo de niñas, el Instituto-Escuela y la Escuela de Párvulos de Simancas. También dependían de la JAE una serie de centros de investigación - algunos nuevos y otros ya existentes - reunidos en torno al Centro de Estudios Históricos y al Instituto Nacional de Ciencias Físico-Naturales.

La política de la JAE a la hora de decidir la concesión de una pensión, según Teresa Marín Eced, no respondía a criterios fijados de antemano sino a unas líneas generales de actuación definidas por los siguientes rasgos: un

\footnotetext{
7 Decreto Fundacional: Real Decreto de 11 de enero de 1907.
} 
espíritu amplio, desprovisto de rigidez y burocratismo; una especial atención a las personas con prestigio profesional; la priorización de temas que respondían a las necesidades del país en ese momento; el apoyo a los temas de investigación de actualidad; un respeto por los informes de las Comisiones de expertos; el impulso constante a la renovación pedagógica, y una especial flexibilidad para adaptarse tanto a los incrementos y disminuciones de presupuesto como a los diversos cambios políticos ocurridos con los años ${ }^{8}$. Dentro de estos rasgos, y a la vista del número de pensiones que concedió a las mujeres, habría que añadir el de mostrar una sensibilidad positiva hacia la promoción femenina, dado que no sólo no discriminó a las mujeres en la concesión de pensiones, sino que, atendiendo a las cifras, las favoreció. Así, en el periodo que va de 1908 a 1919, las jóvenes recibieron el 4\% del total de pensiones, cuando el porcentaje de alumnas universitarias era mucho menor: tan solo el $1.8 \%$ del total del alumnado, en el curso 1915-1916. Esta tendencia se mantendría en el tiempo: entre 1930-1934, ellas recibieron el 13\% de las pensiones, cuando en el curso 1932-1933, las alumnas del conjunto de las universidades españolas, constituían sólo el 6,4\% del total de universitarios 9 .

Estos porcentajes de jóvenes becadas se incrementan, al tener en cuenta otras vías de obtención de becas que beneficiaron a las españolas, y que se explicitan más adelante.

\section{SOLIDARIDAD TRAS LA GUERRA}

En el inicio de la fructífera relación entre universitarias norteamericanas y españolas, que también involucraría a la JAE, y que redundaría en mejoras de la educación superior de las españolas, encontramos como factores concomitantes, además de la tradición misionera arraigada en los Colleges femeninos de la costa Este de los Estados Unidos y la condición de España como país católico, sentimientos ligados a la guerra de Cuba y circunstancias prácticas derivadas de la I Guerra Mundial.

La guerra de Cuba, librada entre España y los Estados Unidos, generó en este último país un deseo de compensar al país perdedor. Al hilo de este sen-

8 MARÍn ECED, Teresa (1990) La renovación pedagógica en España (1907-1936). Madrid, CSIC.

9 Cifras obtenidas a partir de los datos de CAPEL, Rosa María (1986) El trabajo y la educación de la mujer en España (1900-1930), Madrid, Ministerio de Cultura, para las pensiones obtenidas por las mujeres y MARÍN ECED (1990), para el total de pensiones. 
timiento, un Comité de Boston recabaría los fondos necesarios para construir en Madrid el edificio que albergaría el Instituto Internacional (International Institute for Girls in Spain, IIGS), un colegio dedicado exclusivamente a la educación de las mujeres ${ }^{10}$. El IIGS nacía del empeño de Alice Gordon Gulick, como continuidad del Colegio Norteamericano, una institución educativa de carácter misionero protestante, instalado inicialmente en San Sebastián y que durante el periodo de la guerra de 1898, trasladaría su sede a Biarritz. Finalizada la guerra, Alice Gordon Gulick, se propone atraer al Instituto Internacional a las hijas de familias de ideas progresistas y liberales, por lo que el nuevo instituto será aconfesional. Tras la muerte de Gulick, en 1903, los Colleges de Mujeres ${ }^{11}$ de Massachusetts y una liga creada en Boston para recoger fondos, La Liga del International Institut for Girls in Spain, fueron los encargados de convocar reuniones para explicar el proyecto de construcción de un colegio para la educación superior de las mujeres españolas y recoger fondos. En 1905, Carolina Marcial Coronado, alumna española del Instituto Internacional, viajará a los Estados Unidos para hablar de la obra iniciada por el Instituto en este país. El nuevo edificio del IIGS, se abriría en 1910, en Miguel Ángel, 8, Madrid.

Factores como la mala conciencia generada por la guerra, que se salda con la pérdida española de Cuba, Puerto Rico y Filipinas, además del entusiasmo y la red de relaciones de Alice Gordon Gulick, influirán en el apoyo dado al Instituto Internacional para construir el nuevo edificio, un apoyo que se ve como una compensación ofrecida a los vencidos por los vencedores. En un meeting del IIGS, en 1903, se dice:

«Nosotros no somos sus enemigos; somos sus amigos; estamos aquí para vendar sus heridas (...) Vamos a construir esta escuela...no porque consideremos que las muchachas españolas tienen más derecho a la educación que una de Carolina del Sur, de Arizona o de Alaska. No... (sino) porque queremos erigir un monu-

10 La información que hace referencia al International Institute for Girls in Spain (IIGS) está tomada del Archivo del IIGS, depositado en Smith College, Northampton, Massachusetts, USA. Véase también Zulueta, Carmen de (1984) Misioneras, feministas y educadoras, Madrid, Castalia; y ZuLueTA y MORENO (1993).

11 Creados como una alternativa a la intransigencia de las universidades más prestigiosas de los Estados Unidos para admitir a mujeres, las fundadoras, además de fortuna, poseían sólidas ideas religiosas y una profunda convicción de que sus capacidades les hacían merecedoras de una educación superior similar a la del hombre. En ellos se generaría un liderazgo femenino que posibilitó el inicio de un cambio en las relaciones de poder entre los sexos, con repercusiones dentro y fuera de su país. Alice Gordon Gulick, alumna de Mount Holyoke, fue un buen ejemplo. 
mento a la paz (...) Estamos en el momento histórico concreto en el que ese monumento ha de construirse; y decimos que el lugar para erigirlo no es Washington, la capital del país conquistador sino Madrid, la capital del país conquistado (...) Vamos a tomar medidas que tendrán como resultado la construcción en España, en la capital del país, y con el dinero de los conquistadores de aquel país, un monumento de mediación que en los próximos cien años hará de las mujeres españolas lo que las americanas son hoy» ${ }^{12}$.

Entre los que apoyan al Instituto Internacional, ahora ya aconfesional y apolítico, se encuentran el gobernador de Massachusetts W. Muney Grant; el presidente de Harvard, Charles W. Eliot; y los presidentes de las Universidades de Chicago, Darmouth, Mount Holyoke, Wellesley y Smith. El acta de formación de la Corporación bostoniana, bajo la denominación de International Institute for Girls in Spain, acogida a las leyes de la Commonwealth of Massachusetts, se firma en Boston el 5 de Diciembre de 1892. Para el Presidente de la Universidad de Harvard, el IIGS:

«representa un esfuerzo para introducir en España el ideal americano de muchacha y de mujer... Por medio de este Instituto Internacional queremos introducir en ellas (las costumbres) el ideal americano de mujer. Creo que es un ideal de mujer superior al que poseen otras naciones» ${ }^{13}$.

Además, la profesora Gulick y su esposo, casi desde el principio de su estancia en España, establecieron conexiones personales y de amistad con algunos de los hombres de la Institución Libre de Enseñanza, en particular con Cossío y con Azcárate, de los que obtuvieron permanente apoyo y consejo. Esta relación evolucionaría y se plasmaría en una estrecha colaboración con la JAE cuando el Instituto se instala en Madrid, una colaboración que nace de compartir ideas como la libertad de conciencia, el derecho de la mujer a educarse como el hombre y los nuevos métodos pedagógicos ${ }^{14}$.

Años más tarde, esta vez como consecuencia de los problemas de comunicación derivados de otra guerra, la I Guerra Mundial, el Instituto Internacio-

12 Discurso del Rev. Edward Everett Hale, D.D., Boston, Mass. Old South Church. Boston, 25 de enero de 1903. Report of a Public Meeting of the Officers and Friends of the IIGS, pp. 38-39. Archivo del IIGS.

13 Discurso de Charles William Eliot, Presidente de la Universidad de Harvard. Boston, 25 de enero de 1903. Report of a Public Meeting of the Officers and Friends of the IIGS, pp. 40-41. Archivo del IIGS.

14 Zulueta, Carmen de (1992), Cien años de educación de la mujer española. Historia del Instituto Internacional, $2^{\mathrm{a}}$ ed., Madrid, Castalia. 
nal pasaría a estrechar aún más su relación con la JAE, compartiendo edificios y proyectos. Entre otros, con el apoyo del Comité de Boston del Instituto Internacional, en la Residencia de Señoritas se construirá el primer laboratorio de química dedicado en exclusiva a la formación científica de las españolas en este campo, el Laboratorio Foster.

\section{COLABORACIÓN ENTRE LA JAE Y EL INSTITUTO INTERNACIONAL}

La JAE y el Instituto Internacional mantendrán una estrecha relación de colaboración basada, como se ha dicho, en la afinidad de ideas mantenidas por ambos grupos acerca de la educación. Las americanas dejarán su impronta en dos instituciones creadas por la JAE, la Residencia de Señoritas y el Instituto Escuela. Carmen de Zulueta, conocedora estrecha de esta relación, mantiene que la influencia, atribuida a los hombres y espíritu de la Institución Libre de Enseñanza que dan vida a la JAE, fue mutua ${ }^{15}$.

Cuando los problemas generados por la I Guerra mundial van dificultando el transporte de materiales y personas a través del océano, el Instituto Internacional se plantea fundir su acción en los proyectos de la JAE. En 1917, ésta firmará un acuerdo con la corporación de Boston del IIGS por el que alquila las fincas del IIGS para uso de la Residencia de Señoritas. En las cláusulas del contrato se especifica que las profesoras norteamericanas se responsabilizarán de los departamentos de inglés, educación física y laboratorios ${ }^{16}$. A partir de entonces se fusionan las bibliotecas y se posibilitan contactos personales que redundarán en intercambios de alumnas y becas. De hecho el Instituto va a ser una vía abierta, utilizada por la Residencia, de relación con las universidades femeninas norteamericanas, a donde acudirán algunas de las estudiantes españolas más tarde.

En Junio de 1919, Lewis Kennedy Morse —Presidente del IIGS - y Miss Susan D. Huntington — Presidenta del Comité Ejecutivo-; María de Maeztu y José Castillejo, representantes de la JAE y del Ministerio de Instrucción Pública de Madrid, firman otro contrato. En el punto VI se señala, haciendo referencia al edificio de Miguel Ángel 8, que «desde el comienzo de este nuevo contrato el citado edificio deberá usarse solamente para la educación

15 ZuluetA, Carmen de (s.d.) «The Institución Libre and the International Institute for Girls in Spain: cooperation in secular education». Archivo del IIGS, Caja 26, carpeta 696.

16 Agreements between IIGS and the JAE, 1918-24. Archivo del IIGS. El primer contrato está firmado en Madrid, el 1 de octubre de 1918, por Caroline Bourland y Santiago Ramón y Cajal. 
de las mujeres o muchachas ${ }^{17}$. Los contratos de arrendamiento de Miguel Ángel 8 a la JAE se fueron renovando año tras año, siendo el último recogido antes de la guerra, de 1931. Por otra parte, el edificio de Fortuny 53, donde se alojan la mayoría de los servicios de la Residencia de Señoritas, se venderá a la JAE.

\section{LA SOLIDARIDAD E INTERCAMBIOS CON LAS UNIVERSIDADES FEMENINAS NOR-} TEAMERICANAS

Las norteamericanas, ante el rechazo de las Universidades a admitirlas en su seno, fundaron universidades sólo de mujeres, Colleges femeninos; se organizaron para avanzar en sus objetivos y trataron de establecer lazos con mujeres de otros países. Algunas ricas herederas legaron sus bienes para crear estas universidades, y muchas profesoras dedicaron esfuerzos a conseguir que otras lo tuvieran más fácil. La educación y la entrada de las mujeres en las distintas profesiones fue una conquista tenaz y paciente. La solidaridad se materializó en el establecimiento de una política de becas, creadas bajo el impulso de la Association of Collegiate Alumnae (ACA), una organización que en el año 1919 agrupaba a más de diez mil universitarias, entre las que se incluían presidentas, decanas, directoras y catedráticas de todos los estados de la Unión y también de Canadá.

En línea análoga a la señalada antes, de solidaridad tras la guerra, la reflexión del desastre que supuso la I Guerra Mundial, generaría un movimiento internacionalista entre las mujeres educadas. La American Association of University Women, resultado de la unión entre la ACA y la Southern Association of College Women, se unió a organizaciones similares de otros países, para crear la Federación Internacional de Mujeres Universitarias (FIMU). La FIMU, en la que también se integraron las españolas, tenía entre sus objetivos promover la educación de las mujeres en el mundo y fomentar las relaciones de cooperación entre los países.

Las universitarias españolas de los años 20 y 30 del siglo XX, se beneficiaron de esta corriente de solidaridad internacional. Además de las pensiones de la JAE, tuvieron el apoyo de profesoras norteamericanas que, de la mano del IIGS, llegaron a nuestro país para impulsar aquellos campos más deficitarios en la educación de nuestro sistema: los idiomas, las ciencias experimentales y la educación física.

17 Ibídem. 
En Junio de 1919, Martha Carey Thomas, presidenta del Bryn Mawr College de Philadelphia, Pennsylvania, EEUU, y miembro del Comité de Relaciones Internacionales de la ACA, propuso al Ministerio de Instrucción Pública español firmar un acuerdo para intercambiar profesoras y estudiantes entre los Women's Colleges norteamericanos y las universidades españolas ${ }^{18}$. Carey Thomas visita Madrid y se entrevista con María de Maeztu. En respuesta a esta proposición, a mediados de 1920 se constituye en Madrid un Comité para la Concesión de Becas a Mujeres Españolas. Está formado por María Goyri como presidenta, Zenobia Camprubí secretaria, María de Maeztu, la Doctora Arroyo de Márquez y José de Castillejo. Las integrantes del Comité se responsabilizarán de la designación de las becarias, y de ejercer desde España una labor de tutoría. Las becas que gestionará este comité son destinadas a universitarias que desean seguir cursos de especialización en las universidades norteamericanas, cubriendo su importe los gastos de residencia y enseñanza pero no los viajes.

En el momento en que se estudia esta propuesta existía ya un programa de intercambio de becarias, venido de la relación entre la Residencia de Señoritas y el IIGS, entre el Smith College y la Residencia. Este intercambio suponía una asignación de 600 dólares por la parte americana y 600 duros por la parte española, al tiempo que las receptoras de las becas se comprometían a dar 6 horas de español e inglés respectivamente en sus universidades de acogida.

Tras la visita a Madrid de M. Carey Thomas, María de Maeztu plantea a la Junta que se haga cargo del pago de los 600 duros del intercambio con el Smith y que contribuya a sufragar los gastos de viajes de todas las becadas, extrayéndolo «del capítulo de pensiones, toda vez que la Srta. Española que va a América puede considerarse como pensionada» ${ }^{19}$.

Se iniciará así un mecanismo de concesión de becas complementadas que explica el significativo crecimiento del número de estudiosas que viajan a los Estados Unidos a partir de este momento. Hablamos de becas complementadas porque las que recibían una beca de un College norteamericano por esta vía acudían a la convocatoria de pensiones de la JAE para solicitar gastos de

18 La propuesta de la ACA estaba dirigida «To the Ministers of Public Education and the official heads of Educational institutions in Spain, North Africa, Egypt, India, Palestina, Greece, Constantinople, and certain cities in Mesopotamia and Asia Minor». Dirigida al Ministerio de Instrucción Pública es remitida a la JAE con fecha 21 de Junio de 1919. Comité para la concesión de becas a mujeres españolas. Madrid, Archivo de la JAE, 155-46.

19 Escrito de María de Maeztu al Secretario de la JAE, José Castillejo, 1 de junio de 1920. Comité para la concesión de becas a mujeres españolas. Madrid, Archivo de la JAE, 155-36. 
viajes y de estancia que cubriera los días del periodo vacacional en los que se cerraban las residencias que las acogían. Una vez obtenida la beca en el lugar de destino, la Junta aceptaba el correr con los gastos de viaje y estancia parcial correspondientes. En las decisiones de la Junta a este respecto va a jugar un papel importante María de Maeztu, que es en muchos casos quien informa de la conveniencia o no de acceder a la petición. La Residencia de Señoritas se encargaba a su vez de acoger a las profesoras y estudiantes norteamericanas que llegaban a España ${ }^{20}$.

\section{BECADAS EN LOS ESTADOS UNIDOS}

Desde el curso 1920-1921 hasta el de 1935-1936, fueron 31 las españolas becadas en los Estados Unidos, para estudiar o especializarse en distintos temas $^{21}$; más de una, por ser los viajes de la época largos y costosos, permanecería en el país sin regresar en las vacaciones de verano. Al respecto, en una entrevista mantenida en Madrid con Dorotea Barnés, esta química que trabajó en el Instituto Nacional de Física y Química (el Rockefeller), contaba algunos detalles de su vida cotidiana en el verano de 1930, pasado con las profesoras de Smith College. Contaba que la cena era servida en vajilla de plata, y que a ella asistían de traje largo. Pero no era esto lo que le había sorprendido. Lo que realmente le había llamado la atención es que tras la cena, las profesoras y ella misma, ¡fregaban por turno! Y ella, que finalmente y pese a todo, estaba allí porque tenía una beca, pensaba: «iSi me viera mi abuela fregar...!22.

Becadas para estudios relacionados con ciencias experimentales en los Estados Unidos fueron: en el curso 1921-1922: Carmen Castilla, maestra superior e inspectora, en Smith College, para especializarse en «Metodología de la enseñanza de las ciencias»; Concepción Lazarraga, y María Luisa Cañameras, licenciadas en Farmacia, para «Prácticas de química», en Barnard College (New York) y Bryn Mawr (Philadelphia, Pennsylvania), respectivamente; y Loreto Tapia Robson, licenciada en Medicina, para estudios de «Fisiología», en Bryn Mawr. Pilar Claver Salas, profesora del Instituto Escuela y más tarde de la Sección de Ciencias Físicas y Naturales de la Escuela Normal, fue becada para estudios de la «Metodología de la enseñanza de las ciencias», en Vas-

20 Magallón Portolés, Carmen (1998a) Pioneras españolas en las ciencias. Las mujeres del Instituto Nacional de Física y Química, Madrid, CSIC.

21 VAZQueZ RAMIL (2001).

22 Conversación de Dorotea Barnés con C. Magallón, Madrid, 1 de febrero de 1995. 
sar College (Poughkeepsie, New York), en el curso 1926-1927, y en Connecticut College (New London) en 1927-2823.

El Instituto Nacional de Física y Química (INFQ), más conocido por el Rockefeller porque había sido financiado por esta institución norteamericana, a principios de los años 30, acogería las líneas de investigación más prometedoras en el campo de las ciencias experimentales en nuestro país ${ }^{24}$. Organizado en seis secciones, en los cinco años que van de sus inicios al estallido de la Guerra Civil española, en él se integrarían hasta 36 mujeres. En la Sección de Electricidad y Magnetismo, dirigida por Blas Cabrera, Dolores Pardo Gayoso; en la Sección de Rayos X, dirigida por Julio Palacios, Pilar Álvarez-Ude Aguirre, Piedad de la Cierva Viudes y Felisa Martín Bravo; en la Sección de Espectroscopía, dirigida por Miguel A. Catalán, Dorotea Barnés González, Rosa Bernís Madrazo, Ma Paz García del Valle, Josefina González Aguado, Pilar de Madariaga Rojo, Pilar Martínez Sancho y Carmen Mayoral Girauta. La Sección de Química-física, dirigida por Enrique Moles, sería la que concentraría a un mayor número de señoras, hasta 14: Pura Barbero Rebolledo, Adela Barnés González, Asunción Fernández Fournier, María Aragón García Suelto, Amelia Garrido Mareca, $M^{a}$ Luisa Garayzábal Medley, Carmen Herrero Ayllón, Narcisa Martín Retortillo, Carmen Pardo García-Tapia, Carlota Rodríguez de Robles, Concepción Rof Carballo, Pilar Villán Bertrán, Ma Teresa Salazar Bermúdez y Ma Teresa Toral. En la Sección de Química Orgánica, dirigida por Antonio Madinaveitia, hubo tres señoras: Petra Barnés González, Natividad Gómez y Carmen Gómez Escolar. En la Sección de Electroquímica, dirigida por Julio de Guzmán, seis: Patrocinio Armesto, Vicenta Arnal Yarza, Carmen García Amo, Manuela González Alvargonzález, Clara Orozco Barquín y Concepción Zuasti Fernández. De Obdulia de Madariaga y Vicenta Muedra Benedito no sabemos a qué sección estuvieron adscritas ${ }^{25}$.

La mayoría de las mencionadas son alumnas brillantes, pues un tercio del total, 12, tienen Sobresaliente y Premio Extraordinario en la licenciatura; 11 son antiguas alumnas del Instituto Escuela, el centro educativo creado por la JAE a modo de centro piloto o experimento pedagógico, para ser el ejemplo a seguir por el resto de centros de enseñanzas medias. También son mayoría las licenciadas en química, 21, una carrera a veces precedida o compatibilizada con la de farmacia, como es el caso de 4 de las anteriores; licenciadas en físi-

23 Magallón Portolés (1998a).

24 Aunque se inaugura oficialmente en febrero de 1932, los equipos se trasladan y empiezan a trabajar ya en septiembre de 1931.

25 MAGALlÓN PORTOLÉS (1998a), p. 226. 
ca son 6, una de las cuales lo es también en química; 4 son licenciadas en farmacia y de 5 no se poseen datos.

De las 36 mujeres reseñadas son 8 las que en algún momento disfrutaron de una pensión en el extranjero, becadas por la JAE, entre las que se cuentan cuatro de las cinco que más publican. Sólo Teresa Toral, que tiene en su haber un mayor número de publicaciones y que se mantiene en el INFQ de becaria, con seguridad hasta el año 1937 y con alta probabilidad en el 38, no logrará su pensión, pues justamente está en trámites de solicitarla en el año 1936. De estas 8 becadas, cinco lo serán en los Estados Unidos. El cuadro 1, las recoge.

CUADRO N ${ }^{\circ}$ 1. INVESTIGADORAS DEL INFQ BECADAS EN LOS EEUU.

\begin{tabular}{|c|c|c|c|}
\hline $1926-27$ & NOMBRE & College & TEMA DE ESPECIALIZACIÓN \\
\hline & $\begin{array}{l}\text { MARTín BRAVO, Felisa } \\
\text { Doctora en Física } \\
\text { Prof. Auxiliar de Física } \\
\text { en la Universidad Central } \\
\text { INFQ: Sección de Rayos X }\end{array}$ & $\begin{array}{l}\text { Connecticut College } \\
\text { (New London ) } \\
\text { (1927-28) } \\
\text { Middlebury College } \\
\text { (Vermont) } \\
\text { (1932-1933) } \\
\text { (Cambridge, Inglaterra) }\end{array}$ & $\begin{array}{l}\text { Profesora invitada en el } \\
\text { College }\end{array}$ \\
\hline \multicolumn{4}{|l|}{$1929-30$} \\
\hline & $\begin{array}{l}\text { BARNÉS GONZÁLEZ, Dorotea } \\
\text { Doctora en Química } \\
\text { Investigadora en el INFQ } \\
\text { INFQ: Sección de Espectros- } \\
\text { copía }\end{array}$ & $\begin{array}{l}\text { Smith College } \\
\text { Northampton, Mass. } \\
\text { (1930-1931) } \\
\text { Sterling Chemistry } \\
\text { Laboratory, Yale, New } \\
\text { Haven } \\
\text { (1932, Graz, Austria) }\end{array}$ & $\begin{array}{l}\text { Técnicas Espectroscópicas } \\
\text { aplicadas al análisis químico } \\
\text { (Profesora Foster y Profeso- } \\
\text { ra Anslow) } \\
\text { Estudio del ácido nucleínico } \\
\text { (Dr. Coghill) } \\
\\
\text { Espectroscopía Raman } \\
\text { (Prof. Kohlrausch) }\end{array}$ \\
\hline & $\begin{array}{l}\text { INFQ: Sección de } \\
\text { Espectroscopía }\end{array}$ & $\begin{array}{l}\text { Vassar College (Pough- } \\
\text { keepsie, New York) } \\
\text { Standford University } \\
\text { (Palo Alto, California) } \\
\text { (1930-1932) } \\
\text { Columbia University }\end{array}$ & $\begin{array}{l}\text { Estudios de Espectroscopía } \\
\text { y Optica Física }\end{array}$ \\
\hline
\end{tabular}




\begin{tabular}{|c|c|c|c|}
\hline $1931-32$ & NOMBRE & College & TEMA DE ESPECIALIZACIÓN \\
\hline & $\begin{array}{l}\text { GONZÁLEZ ALVARGONZÁLEZ, } \\
\text { Manuela } \\
\text { INFQ: Sección de Electro- } \\
\text { química }\end{array}$ & $\begin{array}{l}\text { Bryn Mawr College, } \\
\text { (Philadelphia, Pennsyl- } \\
\text { vania) }\end{array}$ & $\begin{array}{l}\text { Ampliación de Estudios de } \\
\text { Química }\end{array}$ \\
\hline \multicolumn{4}{|l|}{$1932-33$} \\
\hline & $\begin{array}{l}\text { GARcía Del VALle, Paz } \\
\text { INFQ: Sección de } \\
\text { Espectroscopía }\end{array}$ & $\begin{array}{l}\text { Radcliffe College, } \\
\text { Harvard University, } \\
\text { (Cambridge, Massachu- } \\
\text { setts) }\end{array}$ & $\begin{array}{l}\text { Estudios de Espectroscopía } \\
\text { (Prof. Saunders) }\end{array}$ \\
\hline
\end{tabular}

Fuente: Archivo de la JAE. Elaboración propia.

\section{MARY LOUISE FOSTER}

Entre las profesoras norteamericanas que llegan a España de la mano del IIGS, estará Mary Louise Foster. En 1920, viene a Madrid precisamente para desempeñar el puesto de directora del Instituto Internacional. Esta científica, nacida en Boston en 1865, fundaría en nuestro país el primer laboratorio de química dedicado en exclusiva a la formación de mujeres en este campo. Pionera en muchos aspectos, dedicó toda su vida a la enseñanza y la investigación de problemas ligados a la química, desde los puramente experimentales hasta los históricos, también a impulsar la educación científica de las mujeres, como muestran sus escritos y las opciones vitales que va tomando. Margaret Rossiter la señala como la primera científica de los Estados Unidos que tuvo un empleo en la industria química; se refiere a su trabajo como química en la Standard Essence Company, en Maplewood, New Jersey, en el periodo que va de 1901 a $1904^{26}$.

Graduada en 1883, en la Girls' Latin School de Boston, Foster comienza ese mismo año a dar clase en Chauncy Hall mientras continúa sus estudios para pasar los Boston Examinations. Superadas estas pruebas, pasa a enseñar en una escuela de mayor nivel (grammar grades), la Lowell School, también de Boston. Allí continuará hasta 1888, cuando a sus 23 años decide trasladarse a Northampton, Massachusets, una pequeña ciudad situada a dos horas de Boston, en donde desde 1871 se erige el Smith College, uno de los Colleges

26 Rossiter, Margaret W. (1995), Women Scientists in America Before Afirmative Action, 1940-1972. Baltimore/London, The Johns Hopkins University Press. 
femeninos fundados en la costa Este. Allí llega ya preparada para hacer dos cursos en uno y a la vez ser asistente de laboratorio. Se gradúa en Smith en 1891 y comienza a dar clases de física y química en la West Roxbury High School, de Boston. En esos años, mientras da clases sigue cursos de botánica, fisiología, bacteriología y mineralogía en el Massachusetts Institute of Technology (MIT). En el MIT será alumna de Ellen Swallow Richards, la primera mujer que estudió en esta institución y una de las químicas pioneras de Norteamérica ${ }^{27}$.

En 1899, se traslada a Nueva York, en donde trabaja en distintos laboratorios. En el de Christian Herter, de Columbia (1899-1901), y en el de fisiología del Dr. Philip Hiss (1905-1908). Como se ha mencionado, trabaja en la Standard Essence Company, de 1901 a 1904, y en el curso 1904-1905 es profesora de Química y asistente de investigación en el Woman's Medical College de Nueva York.

En 1908, vuelve al Smith College como profesora del Departamento de Química. Allí permanecerá hasta su jubilación, a mediados de los años 30. A lo largo de los años, en el Smith, disfruta de varios años sabáticos. En uno de estos periodos (1914) se doctora en química, en la Universidad de Chicago. En el verano de 1918, en el Instituto Rockefeller de Investigación médica, Foster contribuye al esfuerzo dedicado a paliar los efectos de la guerra, investigando la kefalina, una de cuyas propiedades es su poder de coagular la sangre. Esta sustancia sería más tarde (hacia 1931) de uso general en forma de vendas de kefalina.

A España llegará en otro de sus sabáticos, en 1920, para desempeñar el puesto de directora del Instituto Internacional. Enseguida se da cuenta de las carencias de formación en técnicas de laboratorio entre las estudiantes de la Universidad de Madrid. Y decide poner en marcha un laboratorio de Química en las dependencias de la Residencia de Señoritas. En 1927 vuelve de nuevo a España, invitada por la Junta y el Instituto Internacional para supervisar el diseño y equipamiento de las nuevas instalaciones del laboratorio de química. Será en la inauguración de estas nuevas instalaciones cuando el laboratorio recibirá su nombre.

Interesada por la historia de la química, asistió como delegada del Smith College al International Congress of History of Science and Technology, celebrado en 1931 en Londres, perteneció a la History of Science Society americana - de la que fue elegida miembro del Council en 1932-, a la American

27 Sobre Ellen Swallow Richard, véase Rossiter (1982). 
Association for the Advancement of Sciences y a la Phi Beta Kappa del Estado de Massachusetts.

La relación de Foster con España se fue intensificando: en los años 20 por su trabajo en el Laboratorio de la Residencia de Señoritas; y en la década siguiente debido a su interés por la tradición histórica de trabajos químicos heredados de los árabes que se suma a su admiración por la riqueza del legado histórico español ${ }^{28}$. En 1934, asistirá al IX Congreso Internacional de Química Pura y Aplicada que tuvo lugar en España. Con este motivo escribe un artículo en el que plasma esta admiración y en el que elogia el trabajo desarrollado por la JAE en beneficio de la educación y la investigación ${ }^{29}$. Además de sus trabajos históricos, las líneas de investigación que cultivó fueron la bioquímica y la espectroscopía molecular, publicando numerosos trabajos. Uno de ellos lo firmaría con Dorotea Barnés, una de sus alumnas más destacadas en Madrid y en Smith College, quien tras dos años de estancia en EEUU, pasó a formar parte del grupo de mujeres investigadoras del Instituto Nacional de Física y Química.

A lo largo de su vida escribió múltiples trabajos sobre diversos temas históricos, entre los que destacan un libro sobre la vida de Lavoisier ${ }^{30}$, otro sobre Bernard Palissy ${ }^{31}$ y la primera traducción al inglés de El Lapidario de Alfonso $\mathrm{X}$ el $\mathrm{Sabio}^{32}$. Este último trabajo será el resultado de una beca que le concede el American Council of Learned Societies para estudiar la Alquimia en España, en el verano de 1930.

Ya jubilada, en la década de 1940, continúa sus investigaciones, ahora sobre proteínas hidrolizadas, al tiempo que el Colegio Americano de Santiago de Chile le encarga la misma tarea que con tanto éxito había llevado a cabo en Madrid: organizar un laboratorio de física y química.

28 Entre sus papeles hay descripciones de Castilla y Extremadura, ensayos acerca de las bibliotecas españolas, de los castillos; también de los trabajos de bordado, heredados de la tradición árabe, y que todavía seguían realizándose en los pueblos españoles.

29 Foster, Mary Louise (1934) «Chemistry in Spain». Journal of Chemical Education, 11(7), 426-427.

30 Foster, Mary Louise(1926) Lavoisier, his Life and Works. «Smith College Monographs», 1, Northampton, Massachusetts.

31 Foster, Mary L. (1945) Bernard Palissy Sixteenth Century Scientist. Boston, Sophia Smith Collection.

32 Foster, Mary Louise y Meyerhoff, Howard A. (1940) The Book of Stones. A Thirteenth Century Manuscript. Traducción de la obra de Alfonso X El Sabio, El Lapidario (Ref.: Smith College, Sophia Smith Collection and College Archives, Caja 834). 
Mary Louise Foster, profesora emérita del Smith College, murió en Pembroke, Massachusetts, el 21 de Junio de 1960, a los 95 años de edad ${ }^{33}$.

\section{El LABORATORIO FOSTER ${ }^{34}$}

Los informes del Instituto Internacional, enviados al Comité de Boston hacen referencia sistemática a la ausencia de prácticas de laboratorio en la formación de las españolas; esta necesidad se detecta con más claridad y se vive con mayor urgencia desde fuera que desde los ámbitos españoles donde se sabe, y se admite con cierta resignación, que la falta de laboratorios es una carencia estructural clásica en España. Habida cuenta de los objetivos que el Instituto Internacional se había marcado - la colaboración en la educación superior de las españolas - , de las estrechas relaciones que mantenía con la Residencia en esos momentos y de los acuerdos que les unían, no es de extrañar que la profesora Foster dedicara gran parte de su tiempo a organizar un laboratorio de química. Para las profesoras y alumnas norteamericanas que viven en la Residencia es inconcebible estudiar química sin hacer prácticas, recurriendo sólo al aprendizaje memorístico.

Ante el requerimiento realizado a través de la directora María de Maeztu, la Junta equipa un local que servirá de laboratorio en la Residencia. Es el laboratorio que más tarde se llamará formalmente Laboratorio Foster. Se trata de un laboratorio universitario, encaminado sobre todo a la adquisición de técnicas básicas, y aunque el nombre es posterior, la puesta en marcha inicial y su crédito pertenecen a Foster ya desde 1920. Desde el principio, los profesores de química de varias facultades ofrecen convalidar las prácticas que se realicen bajo la dirección de la profesora Foster; y el crédito alcanza hasta los últimos años del programa de doctorado. Este respaldo se mantendrá a lo largo de los años e incluso llegará a institucionalizarse en los años 30, cuando las alumnas de los cuatro cursos de Farmacia realizan sus prácticas de química en él. Así lo recuerda Carmen Gómez Escolar, directora del Laboratorio de 1932 a 1936 :

«En la Residencia, en Fortuny 30, teníamos un laboratorio magnífico, en la parte de abajo de la enfermería, a expensas de la JAE. De responsables estábamos dos.

33 Los datos biográficos de M. Louise Foster están sacados del Smith Alumnae Biographical Register, 1935. Smith College, Northampton, Massachusetts, USA.

34 Versión revisada de MAGALLÓN PORTOLÉS (2001). 
Cuando acabé la carrera (a principios de los 30), yo estaba de directora y otra chica (Carmen Sánchez) de auxiliar. Las prácticas que allí hacíamos, de química orgánica sobre todo, eran muy buenas, yo les firmaba el cuaderno y Madinaveitia (profesor de química orgánica en la Facultad) las admitía. Las chicas que las hacían no tenían que hacer examen práctico» ${ }^{35}$.

Los tres primeros cursos, hasta 1923, el Laboratorio Foster estará dirigido por una profesora norteamericana del IIGS. Los dos primeros, será Mary Louise Foster, y el tercero, Vera Colding. El primer año se organizan cuatro secciones y dos cursos, uno de análisis cualitativo y otro de análisis cuantitativo, de cuatro horas semanales. Se cubren todas las plazas ofertadas, unas 30. Durante el verano de 1921 se introducen algunas mejoras, se compran dos nuevas balanzas, se habilita una sala especial para colocarlas y se instala el gas y el agua en la sala dedicada al trabajo cuantitativo. Castillejo, Secretario de la JAE, escribe al Comité de Boston dando cuenta de los avances del laboratorio, cuyo éxito, señala, debe ser motivo de orgullo para la profesora Foster ${ }^{36}$ Así lo cuenta, la propia Foster, años más tarde:

«A pesar de los déficits de equipamiento, que, en ningún sentido, estaba a la altura ni siquiera del más simple y pobre de nuestros laboratorios, prevalecía el entusiasmo y el trabajo duro y aquellas estudiantes, antes de fin de curso, habían adquirido una buena técnica y ciertamente una comprensión del método científico. Muchas de estas alumnas, ahora regentan sus propias farmacias en diferentes ciudades de España...una de aquellas jóvenes es jefe de la sección de vacunas del Laboratorio provincial de Zamora, dos son médicas, una dentista y otra trabaja en los Laboratorios de Aduanas. Es evidente que las mujeres están penetrando en todas las ramas de la vida profesional»» ${ }^{37}$.

En el curso 1921-1922, en el laboratorio se inscriben 43 alumnas, 13 de ellas de Madrid y el resto hasta de 15 diferentes provincias, con un promedio de edad de 19 años. En el informe de la profesora ${ }^{38}$ se indica que la cifra de las madrileñas se ha más que triplicado con respecto a las que asistían el año

35 Conversación con Carmen Gómez Escolar. Realizada y grabada por Santos Casado y Alfredo Valverde el 8 de enero de 1993. Madrid, Centro de Documentación de la Residencia de Estudiantes.

36 Notes and News del International Institute for Girls in Spain, 1, Boston, April 1922.

37 Foster, M. L. (1931) «The Education of Spanish Women in Chemistry». Journal of Chemical Education, 8(1), 30-34, p. 32.

38 Informe de Mary Louise Foster dirigido al Boston Board del IIGS, 25 de mayo de 1922. Archivo del IIGS. Caja 48, carpeta 924. 
anterior. Esto nos da idea de que el Laboratorio atrae alumnas que viven en sus casas de Madrid, fuera de la Residencia. La mayoría son estudiantes de Farmacia, también hay de Química, Medicina, Ciencias y de la Escuela Superior del Magisterio.

La abundancia de estudiantes de Farmacia, la atribuía Foster a la alta estima de las españolas por un trabajo que establecía continuidad con el que otras mujeres, en España, habían realizado en el pasado. Se refería a las monjas, y a su tarea de buscar y cultivar hierbas para administrar a los enfermos en los pueblos. Por otra parte, la Farmacia ofrecía una ocupación retirada del ámbito público, y esto cuadraba con las costumbres.

Metodológicamente, Foster prepara a las asistentes al laboratorio en el desarrollo de la independencia de análisis, la capacidad de iniciativa y la confianza en los resultados, así como en la adquisición de las técnicas adecuadas. En el segundo año, Foster introducirá un curso práctico de química orgánica.

«Para conseguir estos resultados, cada estudiante trabajó independientemente analizando 20 soluciones conteniendo de 3 a 5 metales desconocidos, 25 sales desconocidas y 10 minerales. El curso de segundo año fue de química cuantitativa y en él utilizamos el libro de Casares como texto. Cada estudiante realizó los problemas y practicó todos los métodos (...) Esta destreza se puso en evidencia al acabar el trabajo requerido tres semanas antes de finalizar el cuatrimestre, habiendo aplicado los métodos al análisis de azúcares, aguas oxigenadas y orinas normales y anormales ${ }^{39}$.

Foster encuentra a las alumnas españolas «trabajadoras serias que no regatean esfuerzos pero que no permiten que nada interfiera con su principal objetivo, a saber, aprobar los exámenes de mayo» ${ }^{40}$. Esto hará que la asistencia al Laboratorio se resienta en cuanto se acercan las fechas de exámenes.

La Memoria de la Junta publicada en 1922 proporciona también un informe amplio del Laboratorio, encomiando el trabajo de Miss Foster, del que se dice constituye un modelo de organización a la vez que una muestra de las amplias perspectivas que se abren ante las mujeres en el campo de la Química práctica. El balance de estos dos primeros años será valorado muy positivamente desde distintas perspectivas por la profesora Foster:

«Mi experiencia aquí, una de las más agradables e interesantes de mi vida, me lleva a creer que este trabajo de prácticas de laboratorio es muy necesario, de gran

\footnotetext{
39 Ibídem.

40 Ibídem.
} 
valor y muy apreciado por quienes lo reciben. He tenido la cooperación más generosa y cordial por parte de Miss Maeztu y Mr. Castillejo» ${ }^{41}$.

En estos dos primero años de su estancia en España, en particular en el Laboratorio de su nombre, Foster no parece que se centrara en hacerse cargo de otra de las carencias que a su juicio era importante subsanar en este país, y que ella defendía como un conocimiento estrechamente ligado a la química, a saber, la preparación científica para las tareas domésticas. Fue en el MIT donde ella adquirió esta preocupación por ampliar la ciencia, extendiéndola hacia preguntas más ligadas a espacios tradicionales de las mujeres, línea en la que recibió la influencia de Ellen Swallow Richards. Esta había abierto espacios de trabajo 'femeninos' en la ciencia, al fundar la Economía Doméstica, un campo de gran relevancia en los Colleges de mujeres ${ }^{42}$. En él se abordaban aspectos de la ciencia que hasta el momento apenas habían sido tenidos en cuenta, pues en el caso de la química, ésta se había centrado fundamentalmente en los problemas que interesaban a la industria, entonces concebida como algo sustancialmente aparte del hogar. Entre otros problemas, la Economía Doméstica se hacía cargo del análisis de aguas y de productos alimenticios, así como de la elaboración de dietas equilibradas y saludables. Desde esta nueva perspectiva, pensaba Swallow que la química hacía a las mujeres «mejores amas de casa... y madres más adecuadas para cuidar de la versátil juventud americana $\rangle^{43}$. En coherencia con esta línea había presentado, en la exposición mundial de Chicago de 1893, una cocina «Rumford» que ofrecía a los visitantes comida nutritiva y 'científicamente' cocinada. Mary Louise Foster compartía los criterios de su profesora, también el interés por el nuevo campo acotado por Swallow, la Economía Doméstica, y así lo manifiesta al llegar a España. En este país, escribe, «la necesidad más acuciante, mucho mayor que en Estados Unidos, es la educación de las mujeres en la química y el uso y la preparación científica de comida. Los cursos de ciencia doméstica y economía, y la relación entre la comida y la salud, en mi opinión, son aquí muy necesarios; son, de hecho, el mismo tipo de cursos que la American Chemical Society está poniendo en marcha en los Estados Unidos con la ayu-

41 Ibídem.

42 Para una preocupación similar desarrollada por Rosa Sensat, véase SolsonA, Nuria (1999) «La educación dirigida de las amas de casa. Las aportaciones de Rosa Sensat». En M.J. Barral; C. Magallón; C. Miqueo y M.D. Sánchez (eds.) Interacciones Ciencia y género. Discursos y prácticas de mujeres. Barcelona, Icaria, 233-257.

43 ROSSITER (1982), p. 68. 
da de los clubs de mujeres. Desgraciadamente, no existe una organización similar (a estos clubs) en España $»^{44}$.

Volviendo al Laboratorio Foster, en el curso 1922-23, la directora pasa a ser Vera Colding, graduada en Vassar College, New York (1916), enviada igualmente por el Comité de Boston del Instituto Internacional. Y ya en 19231924, la Junta pone al frente del Laboratorio Foster a Rosa Herrera, licenciada en Farmacia y Ciencias Naturales, que en años anteriores había trabajado como auxiliar de las profesoras Foster y Colding.

En 1925, Castillejo escribe al Comité de Boston dando cuenta del éxito del laboratorio de química y solicitando ayuda para organizar en Madrid, y del mismo modo, es decir, con personal y métodos americanos, un laboratorio de biología. Explica que los graduados en ciencias sufren diariamente la falta de laboratorios de botánica, zoología y física. El Instituto Internacional, por su parte, espera contar con una científica de prestigio para satisfacer al curso siguiente la petición de Castillejo ${ }^{45}$. Desde el Comité de Boston del Instituto Internacional se vislumbran las posibilidades que se abren a las españolas debidamente entrenadas en el campo de la investigación científica, ya que en esos momentos se conoce el acuerdo por el que la Institución Rockefeller va a construir un instituto de Física y Química en Madrid.

«La Junta ha construido un laboratorio de química y promete levantar otro para botánica y zoología. Esperamos que ambos establecimientos puedan ser dirigidos durante años por profesoras americanas, quienes por su parte desean colaborar con la Residencia en la formación de las estudiantes para que aprovechen las oportunidades que tienen en la universidad, en las Residencias y más tarde en los Laboratorios de Investigación de física y química que van a establecerse en edificios que cuestan casi medio millón de dólares, construidos por el Rockefeller General Education Board en un terreno cercano a nuestra propiedad y que la Junta les ha proporcionado para este propósito» ${ }^{46}$.

Las aspiraciones de Castillejo acabarán siendo inviables, ya que antes de crear otro laboratorio diferente será preciso mejorar y asentar el que está en marcha. En diciembre del año 25, las condiciones materiales del laboratorio de la Residencia de Señoritas son insostenibles. Las instalaciones que se

44 FOSTER (1931), p. 33.

45 Notes and News del International Institute for Girls in Spain, 10 (Boston, February 1925). Archivo del IIGS. Caja 48, carpeta 909.

46 Kennedy Morse, Lewis (1926) «Present Conditions and plans for the future». Notes and News del International Institute for Girls in Spain, 11. Boston, September 1926. Archivo del IIGS. Caja 48, carpeta 909. 
habían acondicionado se quedan pequeñas para la demanda existente. Se necesita con urgencia una reparación y una ampliación. No hay otro local disponible, por lo que la JAE comienza la construcción de un nuevo laboratorio de química en el jardín de Fortuny. Las dificultades económicas impiden que las obras se lleven a cabo de manera ágil, con el consiguiente trastorno para la continuidad del trabajo iniciado. Durante el curso 1926-27 tienen que suspenderse los trabajos por falta de fondos y María de Maeztu viaja a Boston, donde consigue de la Corporación del IIGS un donativo de veinte mil pesetas para continuarlas. Consigue también que, en Junio de 1927, la profesora Foster vuelva de nuevo con un sueldo pagado a medias por el IIGS y por la JAE para equipar y poner en marcha el nuevo Laboratorio de química. Ya en Madrid, Foster supervisa el diseño y equipamiento de las nuevas instalaciones y trata de que el nuevo recinto se acerque lo más posible a Stoddard Hall, el laboratorio de Química del Smith College. El laboratorio, que tiene dos dependencias habilitadas, una para análisis cualitativo, con 22 plazas y otra para análisis cuantitativo, con capacidad para 10 personas, se abrirá en enero de 1928, con la consiguiente alegría de las alumnas.

Al nuevo laboratorio asistirán treinta alumnas que básicamente llevarán a cabo el programa ya establecido: prácticas de química inorgánica y de química orgánica. La directora, de nuevo Mary Louise Foster, es ayudada en su trabajo por las profesoras Rosa Herrera y $\mathrm{M}^{\mathrm{a}}$ Luz Navarro. El 1 de marzo de 1928, María de Maeztu, las estudiantes del laboratorio y las profesoras de la Residencia preparan una sorpresa para Mary Louise Foster. A las 4 de la tarde se reúnen en la puerta de entrada del laboratorio y María de Maeztu les dirige la palabra:

«Estamos aquí reunidas para dedicar nuestros nuevos laboratorios de química. Han sido construidos con dinero americano y los cursos han sido fundados y organizados por una americana. En los Estados Unidos la costumbre es perpetuar la memoria de tales servicios dando el nombre del fundador al edificio. Así, para que las que os sucederán en los años venideros se familiaricen con el nombre de esta profesora, este laboratorio se llamará 'Laboratorio Foster'» ${ }^{47}$.

Al terminar su discurso María de Maeztu descubre una placa de bronce colocada en la pared del edificio del laboratorio con un nombre impreso: Laboratorio Foster.

47 Discurso de María de Maeztu, incluido en Foster, M. Louise (1928-29) «An American Laboratory in Spain». Smith Alumnae Quarterly, 20, 45. Véanse también las Memorias de la JAE, 1926-1928. Madrid, 1929, p. 352. 
Durante el curso de 1928-1929, asisten al Laboratorio 32 alumnas, y es dirigido de nuevo por Rosa Herrera, teniendo por ayudante a María Luz Navarro. En el curso 1929-1930, en el que la directora, Rosa Herrera, disfruta de una beca de la JAE para estudios de química en Inglaterra, el Laboratorio Foster pasa a ser dirigido por el profesor Enrique Raurich, de la Facultad de Farmacia, siguiendo de ayudante María Luz Navarro.

Las Memorias de la JAE recogen los trabajos llevados a cabo. Mencionaremos algunos de ellos: «Las señoritas Dolores Quesada, Isabel Carrión y Consuelo Ochoa, asistieron asiduamente y con verdadera aplicación, resolviendo seis problemas de Análisis químico cualitativo, incluso alguno que constituía el llamado «caso complicado». Al empezar los trabajos de Análisis cuantitativo estas mismas señoritas se prepararon y comprobaron las soluciones valoradas más corrientes, verificando repetidas veces, hasta imponerse bien de la técnica, las siguientes determinaciones cuantitativas: glucosa, cloruros y fosfatos en la orina; acidez total, fija y volátil; grado alcohólico, tanino y sulfatos en los vinos; número de acidez, de saponificación, de yodo, de acetato; residuo insaponificable, falsificaciones y adulteraciones en un aceite traído por una de las señoritas, materia orgánica y cloruros del agua del Laboratorio (...) La señorita María Luz Navarro, en funciones de ayudante, prestó con asiduidad y competencia valioso concurso y ayuda aprovechando los momentos libres para empezar a trabajar en análisis de quesos (...) El señor Raurich, gracias al buen orden observado por todas las alumnas, pudo comprobar y arreglar una marcha sistemática para la separación cualitativa e identificación de los ácidos inorgánicos más corrientes. Este trabajo se presentó en las sesiones que del 1 al 4 de mayo de 1930 celebró en Sevilla la Real Sociedad Española de Física y Química, con motivo de su primera reunión anual» ${ }^{48}$.

En el curso siguiente, 1930-1931, la dirección del laboratorio vuelve a manos de Rosa Herrera, manteniéndose María Luz Navarro de auxiliar. Asisten 34 alumnas de la Residencia. En los últimos años el Laboratorio Foster fue dirigido por la citada Carmen Gómez Escolar, auxiliada por Carmen Sánchez. El cuadro $\mathrm{n}^{\circ} 2$ incluye las distintas directoras y el número de alumnas, año a año.

48 Memorias de la JAE correspondientes a 1928-1929 y 1929-1930. Madrid, 1931, pp. 397-400. 
CUADRO N ${ }^{\circ}$ 2. Directoras Y N $^{\circ}$ DE ALUMNAS DEL LABORATORIO FOSTER

\begin{tabular}{|c|c|c|}
\hline Curso & DIRECTORA & NÚMERO DE ALUMNAS \\
\hline $1920-1921$ & Mary Louise Foster & 30 \\
\hline 1921-1922 & Mary Louise Foster & 43 \\
\hline $1922-1923$ & Vera Colding & 19 \\
\hline 1923-1924 & Rosa Herrera & 30 \\
\hline 1924-1925 & Rosa Herrera & $?$ \\
\hline $1925-1926$ & Se cierra & - \\
\hline 1926-1927 & Continúa cerrado & - \\
\hline $1927-1928$ & Mary Louise Foster & 30 \\
\hline 1928-1929 & Rosa Herrera & $?$ \\
\hline 1929-1930 & Enrique Raurich & $?$ \\
\hline 1930-1931 & Rosa Herrera & 34 \\
\hline 1931-1932 & Rosa Herrera & $?$ \\
\hline $1932-1933$ & Carmen Gómez Escolar & 42 \\
\hline 1933-1934 & Carmen Gómez Escolar & 39 \\
\hline 1934-1935 & Carmen Gómez Escolar & $?$ \\
\hline
\end{tabular}

Fuente: Memorias de la JAE y otros documentos. Elaboración propia.

La trayectoria de muchas alumnas del Laboratorio Foster pasaría más tarde por el Instituto Nacional de Física y Química (INFQ). Ambas eran instituciones creadas y gestionadas por la JAE y estaban entrelazadas por una red común de personas. Así, por ejemplo, Blas Cabrera, director de la Sección de Electricidad del INFQ, era vocal del Comité que regía la Residencia. En cuanto a las alumnas, mencionaré tres ejemplos de mujeres que, estando ligadas a la Residencia, trabajarían más tarde en el INFQ. Una es Dorotea Barnés González $^{49}$, que vivía con su familia en Madrid pero asistía a las clases del Laboratorio Foster, a través del que se siente ligada a la Residencia de Señoritas. Otra es Felisa Martín Bravo, que vivió en la Residencia, siendo una de las becarias de la misma a mediados de los años 20 (1924-1926); más tarde sería también profesora de física en ella, profesora auxiliar en la Universidad Cen-

49 Sobre Dorotea Barnés véase Magallón PortolÉs, Carmen (1998b) «Dorotea Barnés y la Espectroscopía Raman». En: J.L. García Hourcade, J.M. Moreno y G. Ruiz (coord.) Estudios de Historia de las Técnicas, la Arqueología Industrial y las Ciencias. Salamanca, Junta de Castilla y León, 2 vols., 817-826. 
tral de Madrid e investigadora en la Sección de Rayos X del INFQ. También fue residente Pilar Martínez Sancho, que trabajó con Miguel A. Catalán en la Sección de Espectroscopía del INFQ.

\section{EPÍLOGO}

Tras la Guerra Civil de 1936, las instituciones que había creado la República fueron desmanteladas y los equipos de investigación se deshicieron. La mayoría de los hombres y mujeres de ciencia, como tantos otros, tuvieron que exiliarse. Todos tuvieron difícil continuar desarrollando sus investigaciones en los países que les acogieron, pero para ellas la ruptura sería en muchos casos definitiva.

El Comité de Boston continuó prestando apoyo a los amigos. Envió ayuda a los refugiados en Francia y a quienes recalaron en Méjico. Lo mismo hicieron las profesoras norteamericanas que habían estado en algún momento en el Instituto Internacional, en España; es el caso de Margaret Palmer que se dedica a apoyar a los refugiados españoles que llegan a París. El edificio de Miguel Angel, 8, que continuaba siendo propiedad americana, terminada la guerra fue reclamado por el Gobierno de los Estados Unidos, pero no se devolvió hasta 1944.

En 1944, Enriqueta Martín, que tras la guerra sigue en Madrid a cargo de la Biblioteca del Instituto Internacional escribe al Comité de Boston:

«Parecía antes que no existía peligro de expropiación o, en todo caso, que podría haber alguno remoto. Ahora se ve que, por el contrario, la amenaza existe y que será necesario actuar con energía y rapidez. El Sr. Embajador viéndolo también así, ha gestionado la rescisión del contrato con el Ministerio de Asuntos Exteriores (...) Duele ver que el Instituto Internacional presta su ayuda económica a una cosa que no aprovecha verdaderamente en el sentido de los motivos para los que se hizo la fundación (...) Es también insoportable que las decisiones del Instituto Internacional o de quienes le representan sean una vez y otra desatendidas y menospreciadas: en 1939 se recibió orden de venir a separar la Biblioteca del Instituto Internacional. Eulalia, actuando de directora provisional se negó en redondo, con el apoyo de Julio Palacios (hoy Vicerrector de la Universidad y encargado de los Colegios Mayores). Más tarde, cuando Mrs. Vernon ordenó que se diese en la Biblioteca un té en honor del Sr. Embajador, fue Matilde quien se opuso rotundamente; cuando el Instituto Internacional consideró que debía terminar el contrato, el Ministerio ni siquiera se dignó acusar recibo de la carta en que se anunciaba y fue necesario exigir un documento que acreditase, al menos, la entrega de aquella (...) Pensándolo bien, todas estas cosas y un sinnúmero de pequeños detalles que, reflexionando, se pueden percibir, se ofrecen como manifestaciones de una intención 
de ir prescindiendo cada vez más del propietario de la casa hasta llegar a anularlo; y entonces sería tiempo de declarar de derecho lo que ya se realiza de hecho.

He aquí, pues que evidentemente ha llegado el momento de librarse de los inquilinos por cualquier medio que sea eficaz y rápido (...) $\rangle^{50}$.

Los boletines del International Institute for Girls in Spain, que edita el Comité de Boston dan noticia del destino y actividad de algunas de las alumnas de la Residencia durante la guerra: de Pilar de Madariaga se dice que está dedicada al trabajo en un orfanato, en Alicante, que se le ha invitado a dar clases en Vassar College pero ella ha rehusado, alegando que en esos momentos (1937) es más necesaria en España ${ }^{51}$. De Dorotea Barnés se dice que continúa, en 1938, dando clases en la Escuela Normal para chicas, de Carcassone, Francia, mientras Arsenia y Justa Arroyo que habían llegado a Bryn Mawr y Smith College, respectivamente, en 1937 han recibido una nueva beca para que continúen estudiando en los Estados Unidos ${ }^{52}$. Uno de los boletines incluye una carta que envía Rosa Herrera desde la Colonia de Refugiados españoles de Villefranche-de-Rouergue (Aveyron). Acabaré con el texto de esta carta que ejemplifica crudamente el destino de esta generación.

«Estoy en Francia al cargo de un grupo de 26 niños españoles de 12 a 16 años, del Instituto de Segunda Enseñanza de Manresa, en donde yo era profesora de Ciencias Naturales en el momento que comenzó el desastre (...) Las autoridades catalanas nos ordenaron evacuar a los niños internos para salvarlos de los peligros de una ciudad sitiada, así es que nos dirigimos a la carretera de Olot. Nuestra intención era trasladar el Instituto a algún pueblo de los Pirineos pero los sucesos ocurrieron tan rápidamente que, con la pérdida de Cataluña casi en 15 días, no hubo tiempo para organizar nada y tuvimos que cruzar el Pirineo a pie, en la madrugada, tras una noche heladora que pasamos cerca de la frontera, en el corazón de las montañas. Para entonces éramos sesenta y cinco, y treinta de los niños eran de 4 a 7 años, algunos incluso más jóvenes. Finalmente cuando esto acabó nos esperaba otra tragedia mayor: la de encontrarnos en un país extranjero, los niños sin ropa, pues hubimos de abandonar todo el equipaje.... ${ }^{53}$.

50 Carta de Enriqueta Martín al Comité de Boston del IIGS, 22 de febrero de 1944, Archivo del IIGS, Caja 48, carpeta 925, Smith College.

51 Notes and News, International Institute for Girls in Spain, number 23, October, 1937.

52 Notes and News, International Institute for Girls in Spain, number 24, October, 1938.

53 Notes and News, International Institute for Girls in Spain, number 25, June, 1939. 